\title{
How to Assured Quality in a Cloud and its Verification
}

\author{
Process \\ Prince Jain \\ Lecturer, Malwa Polytechnic \\ College, Faridkot, Punjab, \\ India
}

Dr. Gurdev Singh

Professor, Gurukul

Vidyapeeth, Banur Punjab, India.

\author{
Dr Mandeep Singh \\ Associate Professor, \\ Chandigarh University, Punjab, \\ India
}

\begin{abstract}
Cloud computing is discipline which use everything as service that provide economic, convenient and on-demand services to requested end users and cloud service consumer. Building a cloud computing network is not an easy task. It requires lots of efforts and time. For this, there arises a concept called Cloud Engineering. Cloud engineering is a discipline that uses set of processes which help to engineer a cloud network. The structure and principles of cloud engineering plays an important role in the engineering of usable, economic and vibrant cloud. The cloud engineering use a cloud development life cycle (CDLC) which systematic developed cloud. Quality assurance and verification is an important and mandatory part of development cycle. Quality assurance ensures the quality and web service of cloud network. Cloud Verification is an irrespirable step in a development of an economic cloud computing solution of a network. Verify the performance, reliability, availability, elasticity and security of cloud network against the service level agreement with respect to specification, agreement and requirement. The work in this paper focuses on the Quality Assurance factors and parameters that influence quality. It also discuses quality of data used in a cloud. This paper proposes and explores the structure and its component used in verification process of a cloud.
\end{abstract}

\section{General Terms}

Cloud, Cloud Computing.

\section{Keywords}

Cloud Quality, Cloud Verification, Cloud Quality Parameters, Cloud Quality Factors.

\section{INTRODUCTION}

Cloud computing is a model for enabling convenient, ondemand network access to a shared pool of configurable computing resources that can be rapidly provisioned and released with minimal management effort [1] [2]. In simple words, the Cloud Computing is combination of a technology, platform, hosting provider, storage services provider on the Internet [3]. The aim of the cloud computing is to provide scalable and inexpensive on-demand computing infrastructures with good quality of service levels [4] [5]. To successful implementation of cloud computing system, a cloud engineering discipline is used.

Cloud Engineering is a discipline that uses both the business and technology thinking for purposes of engineering a cloud [6]. For engineering a cloud, there is very important to follow a model. Cloud development life cycle (CDLC) is the one of the available component model used in the structure of the cloud engineering [7]. CDLC is the iterated, linear organized, isolated executable and flexible life cycle model for the systematic and scientifically development, deployment and delivery of cloud. CDLC contains six phase: Understand Requirement and Analysis; Architect; Implementation and Integration; Quality Assurance and Verification; Deploy, Testing and Improvement; Monitor, Migrate and Audit. The phase Architect; Quality Assurance and Verification; Deploy, Testing and Improvement; Monitor, Migrate and Audit have given its feedback to Understanding Requirement and Analysis phase [7]. The Quality assurance and verification is related to ensure the quality and web service of cloud network; and verify the performance, reliability, availability, elasticity and security of cloud network at the service level against specification, agreement and requirement [7].

\section{HOW TO ASSURANCE CLOUD QUALITY}

Quality assurance in cloud computing ensures the quality of cloud network with the help of audit the cloud. Audit a cloud means measuring the performance of the cloud computing system with the help of compliance, regulation, security, governance and risk of a cloud [8].

Quality of a cloud plays an important role in the performance of cloud network. As it becomes a crucial step in which cloud service provider have to be assured about the quality of a cloud demanded by end user [8]. By ensuring a quality in a cloud gives significant administrative and operational benefits to end user and sometime it becomes a necessary step when end user use public cloud or Internet because firstly in the case of public cloud, cloud services and resources are available publicly, openly and use many times. Secondly, cloud quality in public cloud is very difficult and near to out of control. One of the solutions for such problem is to implement a private cloud that gives virtualization and public cloud technology while keeping resources and services within its control. Another way to deal with it is to implement a hybrid cloud which means some resources placed within the network and some are accessed in the cloud.

Cloud quality is the combination of availability, performance, security and privacy that ultimately will inspire end users to adopt cloud computing network which ultimately save cost. Parameters of cloud quality arranged and are shown in figure1. 


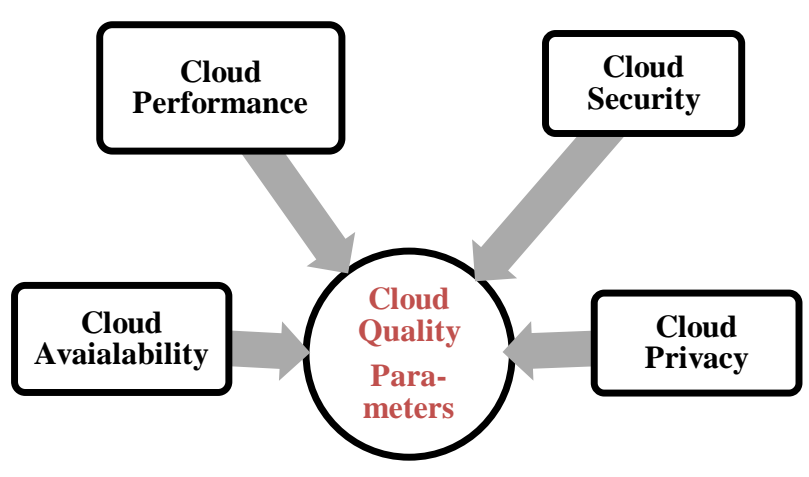

Figure1: Cloud Quality Parameters

Cloud availability is an important parameter which assures availability of cloud on-demand and on-time. This is directly propositional to quality of a cloud. High availability means high cloud quality. Cloud performance is an attribute of quality which measures the performance of cloud whenever their services are in use. Cloud performs according to consumer needs and usage. Cloud security and privacy related to the data, resources and services used by the cloud service consumer.

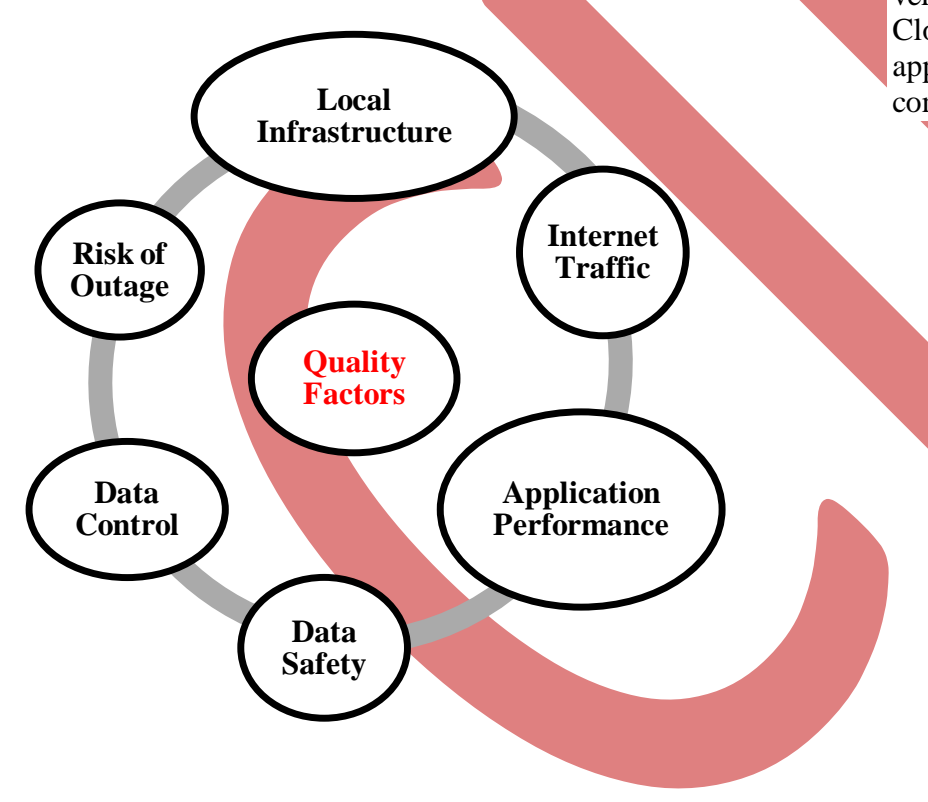

Cloud Quality assurance provides assurance to all client, end user, service consumer and stakeholders that their privacy and security requirements are met. Quality of a cloud depends upon many factors such as quality of local infrastructures, Internet traffic, application performance, data safety and control, and risk of outages as shown in figure- 2 below.

\section{Figure2: Cloud Quality Factors}

Securing the data is a serious and tedious task. It requires lots of investment in form of time and cost. Any organization needs the accurate, consistence, clean data which becomes a base for decision making about cloud consumer. It is also important to maintain quality of precious data for an organization to be used. For this, there are many data quality solution are available which improves the accuracy and timeliness of the data and information related to an organization.

\section{CLOUD VERIFICATION PROCESS}

Cloud Verification is an important and necessary step in a development of an economic and on demand cloud computing solution of a network. For proper verification of cloud is to be done, there is strictly need for a structure to be present. Cloud verification structure is the combination of four components: Cloud evaluation, Cloud Adoption, cloud security and cloud application. These components are further divided into subcomponents and are well explored in figure-3. 
Cloud Verification

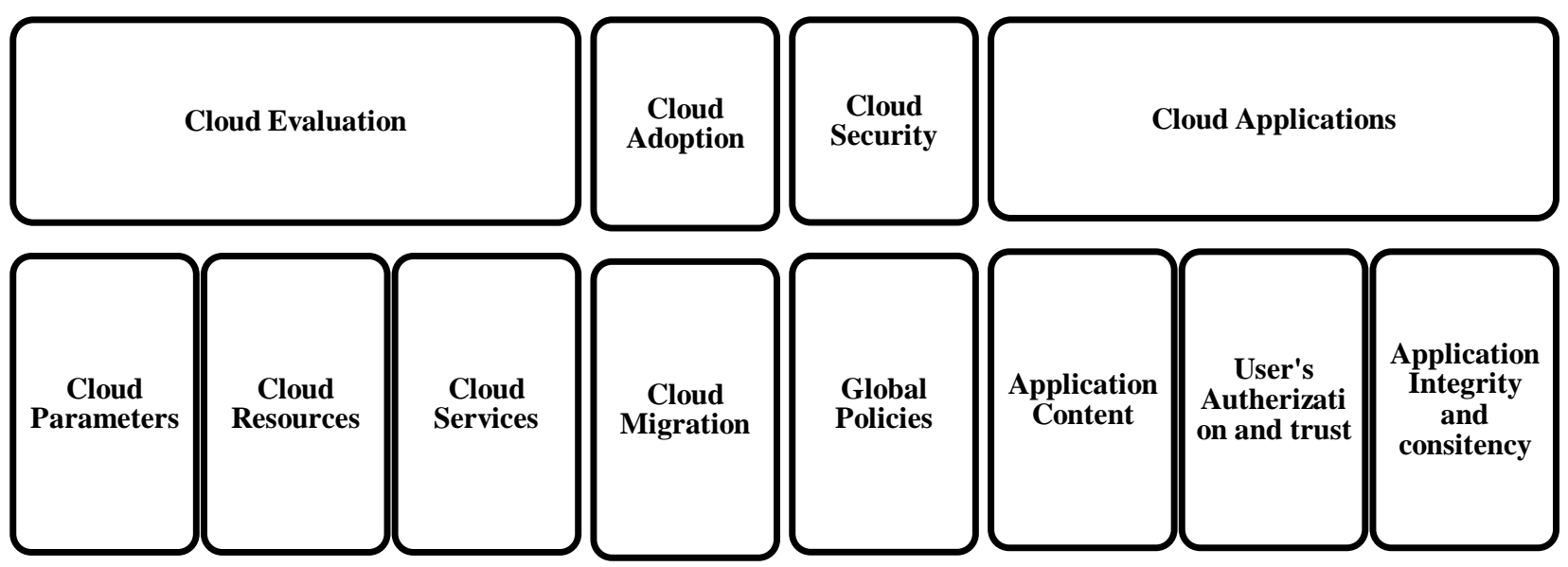

Figure3: Cloud Verification Structure

Cloud evaluation is the evaluation of all cloud parameter, resource and services so that it should be available on demand and on-time to the end user. These evaluated cloud parameters, resources and services are compared against specification, agreement and requirement of service level agreement (SLA). Cloud adoption verification is the adoption of a cloud computing solution of a network by an end-user. Cloud Security verification is the verification of the global security policy of corporate after the migration is consistently preserved. Cloud applications verification is the verification of user's trust, verification of user's authorization, verification of application content or information according to the specific security requirements of different applications. Cloud applications verification also involved verification of operation consistency. It verifies cloud integrity or consistency of an application and gives notification when these are violated.

Password based protection is not enough and suitable in cloud security as passwords may easily be phished, hacked, cracked and reset. Hence, there should be another layer of verification like two factor authentication that is user will be asked to present two different forms of authentication.

Cloud computing verification is not one time process. It is done at random manner after some fixed time. Cloud computing verification is become necessary and required step whenever resources and services are placed in distributed manner. Cloud computing solution verification can also be done on-demand solution of a client or user. This solution provides the flexibility to add, remove and modify computer resources to meet verification demands.

\section{CONCLUSION}

A cloud is relative a new discipline and in a future it will play an important role in everyone lives, but to get there, there is need to assures quality of a cloud. To assures quality there are many parameters and factors which is to be satisfied. This research area needs lots of research in future and can open many new research areas related to it. In the future scope, the other phase of the cloud development life cycle such as Architect; Implementation and Integration; Deploy, Testing and Improvement; Monitor, Migrate and Audit can be explored and require lots of research.

\section{References}

[1] http://searchvirtualdatacentre.techtarget.co.uk/news/ 1510117/Community-cloud-Benefits-and-drawbacks

[2] Michael glas and Paul Andres, OCT 2010, An Oracle white paper in enterprise architecture- achieving the cloud computing vision, CA-U.S.A.

[3] Harjit Singh Lamba and Gurdev Singh, July 2011, Cloud Computing-Future Framework for e-management of NGO's, IJoAT, ISSN 0976-4860, Vol 2, No 3, Department Of Computer Science, Eternal University, Baru Sahib, HP, India.

[4] Dr. Gurdev Singh, Shanu Sood, Amit Sharma, June 2011, "CM- Measurement Facets for Cloud Performance", IJCA, Volume 23 No.3, Lecturer, Computer science \& Engineering, Eternal University, Baru Sahib (India).

[5] Joachim Schaper, 2010, Cloud Services, 4th IEEE International Conference on DEST, Germany.

[6] Stefan Tai, 2009, Cloud Service Engineering, 18th IEEE International Workshops on Enabling Technologies, Karlsruhe Institute of Technology (KIT) and FZI Forschungszentrum Informatik, Karlsruhe, Germany.

[7] Gurdev Singh, Gaurav Garg, Prince Jain, Harmandeep Singh, Nov 2011, The structure of cloud engineering, International Journal of Computer Applications 33(8):44-49, New York, USA.

[8] http://www.channelinsider.com/c/a/Commentary/CloudComputing-Needs-Better-Quality-Assurance-130814.html 